\title{
GENETIC ANALYSIS OF A POPULATION OF TRIBOLIUM III. FATTY ACID COMPOSITION OF UNSATURATED FATTY ACID SENSITIVE MUTANT*
}

\author{
R. F. COSTANTINO, R. O. MUMMA and T. E. BRUSZEWSKI \\ Departments of Biology and Entomology, Pesticide Research Laboratory and \\ Graduate Study Center, Pennsylvania State University, University Park, Pa., \\ U.S.A.
}

Received 3.x.69

\section{INTRODUCTION}

Costantino, Bell and Rogler $(1966,1967,1968)$ isolated and described the phenotype of an autosomal recessive gene in the flour beetle Tribolium castaneum whose biological function is associated with lipid metabolism. Several properties of this mutant homozygous corn oil sensitive (cos/cos) genotype were revealed. The mutant and normal $(+/+)$ genotypes are similar when grown on diets devoid of vegetable oils or unsaturated fatty acids. However, in the presence of the latter two materials the mutant genotype's growth rate and viability are markedly reduced. On diets containing oleic, linoleic and linolenic acids the expression of the mutant genotype is clearly associated with the degree of unsaturation and the concentration of the fatty acid in the diet. The normal $(+/+)$ genotype was not altered by these fatty acids nor, interestingly, was the mutant genotype when stearic or palmitic acids were ted.

These data suggested that the mutant genotype may not be able to metabolise unsaturated fatty acids in a normal manner and that perhaps a toxic metabolite of the unsaturated fatty acids was accumulating, resulting in an altered growth pattern. Therefore, it seemed reasonable to examine the fatty acid composition of the mutant $(\cos / \cos )$ and normal $(+1+)$ genotypes of $T$. castaneum when grown on diets with and without corn oil (61.9 per cent. linoleic acid) in the hope of gaining some insight into the biochemical defect.

\section{Materials and methods}

(i) Insects and culturing techniques

Two genetically different populations of $T$. castaneum were examined in this study: the normal $(+/+)$ or wild type genotype and the mutant corn oil sensitive $(\cos / \cos )$ genotype. The history of these stocks was discussed in our previous papers.

Eggs of each genotype were cultured on the two diets described in table 1 and maintained in a controlled chamber at $33^{\circ} \mathrm{C}$. and 65 per cent. relative humidity. Insects were collected from each culture every other day from 10 days of age through the 28 th day. Due to the very small size of the early larval stages, 200 larvae were collected for lipid extractions from each treatment on days 10 and 12; subsequently, 100 insects were collected for

* This work was supported in part by the U.S. Public Health Service Grant AM 08481. Journal Paper No. 3621 from The Pennsylvania Agricultural Experiment Station. 
lipid extraction. An additional 25 organisms were collected from the cultures for wet and dry weight determinations.

\section{(ii) Extraction procedure}

Larvae, pupae, and adults were placed in $12 \mathrm{ml}$. centrifuge tubes and $2 \mathrm{ml}$. of chloroform-methanol $(1: 2 \mathrm{v} / \mathrm{v})$ were added. The samples were crushed with a glass stirring rod and extracted twice for 20 minutes at $38^{\circ}$. After each extraction the mixture was centrifuged for 3 minutes at 500 r.p.m. The extracts were separated from the sedimented debris and combined. The combined extracts were placed in tared centrifuge tubes and evaporated to dryness under nitrogen. The tubes were then placed in a vacuum for $\mathbf{5}$ minutes and the total extract weight determined.

TABLE 1

\begin{tabular}{lcc}
\multicolumn{3}{c}{ Composition of diets } \\
\multicolumn{1}{c}{ Content } & Diet 0 & Diet 4 \\
Wheat flour & 89 & 89 \\
Yeast & 5 & 5 \\
Glucose. $\mathrm{H}_{2} \mathrm{O}$ & 6 & 2 \\
Corn oil & 0 & 4
\end{tabular}

The crude lipid extracts were dissolved in $1.5 \mathrm{ml}$. chloroform-methanol $(2: 1 \mathrm{v} / \mathrm{v})$ and washed according to Folch, Lees and Sloane-Stanley (1957). The chloroform phase was transferred to vials, evaporated to dryness under nitrogen, placed in a vacuum for 10 minutes and weighed. The lipid extract was then dissolved in $1 \mathrm{ml}$. of chloroform and stored at $-33^{\circ}$.

For dry and wet weight determinations, 25 organisms were placed in tared vials, weighed, and dried in an oven at $110^{\circ}$ for 25 hours, and weighed again.

\section{(iii) Preparation of methyl esters}

A portion of lipid extract $(0.3 \mathrm{ml}$.) was evaporated to dryness in a $2 \mathrm{ml}$. vial and $0.1 \mathrm{ml}$. benzene and $0.4 \mathrm{ml}$. of 12.5 per cent. boron trifluoridemethanol were added (Morrison and Smith, 1964). The vial was tightly sealed with a teflon-lined screw cap and placed in an oven at $110^{\circ}$ for 45 minutes. After the vial cooled, the solution was transferred to a centrifuge tube and 1 volume of $\mathrm{H}_{2} \mathrm{O}(0.5 \mathrm{ml}$.) and 2 volumes of n-pentane $(1.0 \mathrm{ml}$.) were added. The centrifuge tube and its contents were shaken and centrifuged. The upper n-pentane phase was transferred to another vial and evaporated under nitrogen. The methyl esters were redissolved in a minimal volume of $n$-pentane prior to gas chromatography.

\section{(iv) Gas chromatography}

The methyl esters were analysed by gas chromatography (Micro Tek Model 220) utilising a 6-foot 15 per cent. diethylene glycol succinate column at $180^{\circ}$. Methyl esters were identified by comparison of their retention times with those of standards (Supelco, Bellefonte, Pa.). Quantitative measurements of the methyl esters were made using an Aerograph 471 digital integrator. 


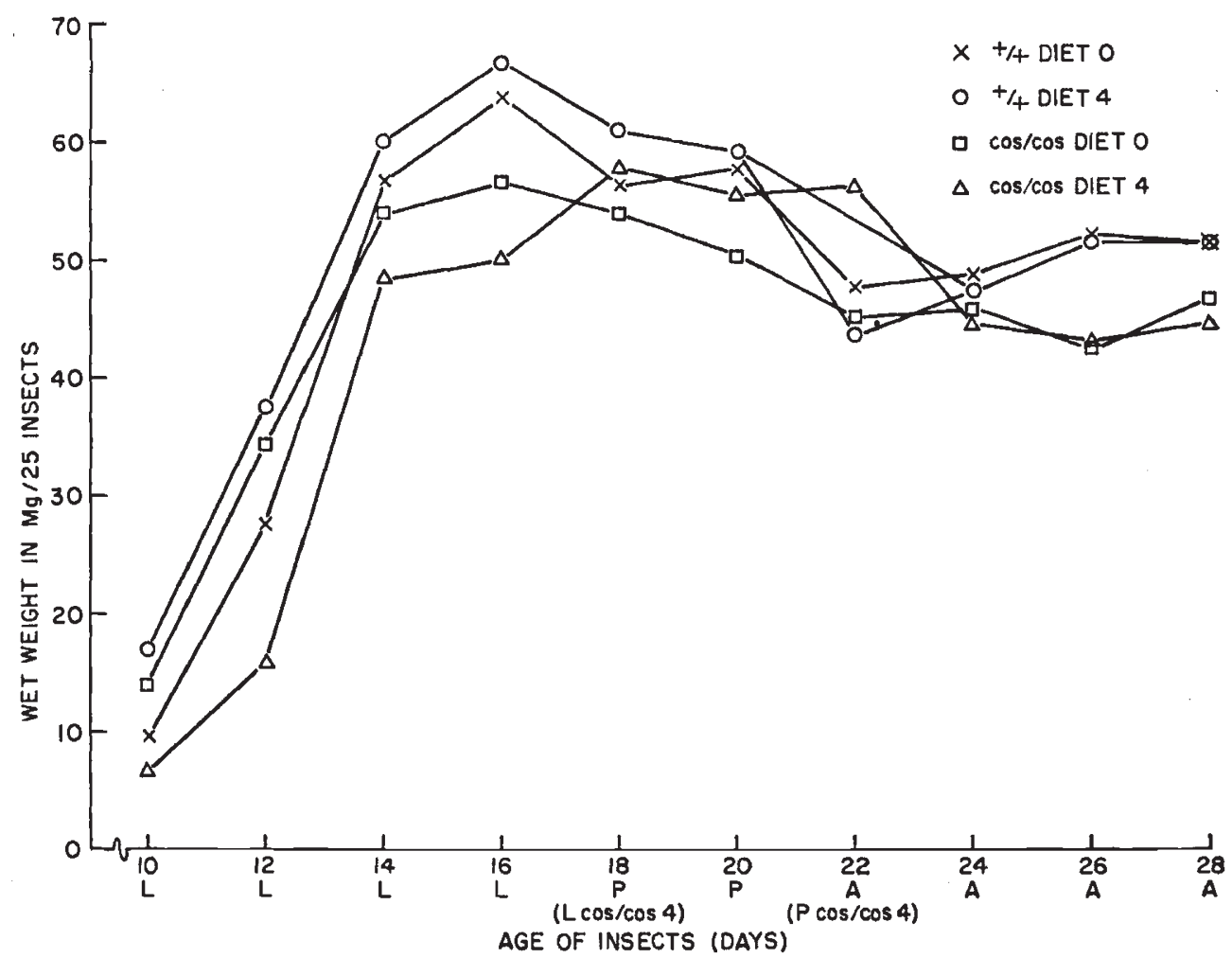

FIg. 1.-Wet weight of experimental groups from 10 to 28 days.

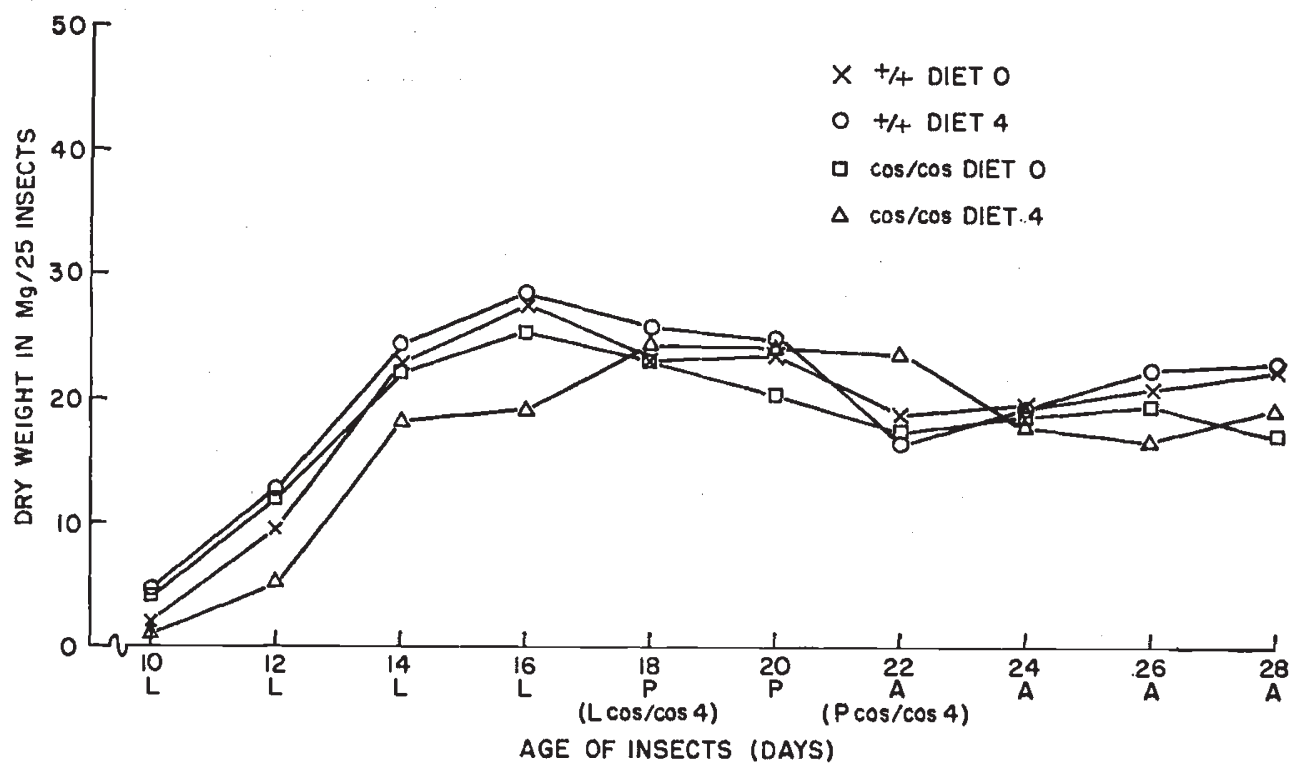

Fig. 2.-Dry weight of experimental groups from 10 to 28 days. 


\section{RESUlTS AND DISCUSSION}

The mutant genotype (cos/cos) has been characterised by displaying an altered growth pattern in response to the concentration and degree of unsaturation of fatty acids present in its diet. The normal genotype $(+1+)$ is essentially unaffected by these variables. Our objective in the following studies was to investigate the possibility of differential utilisation of fatty acids in these two genotypes. In addition to determining the relative percentages of fatty acids, wet weight, dry weight, per cent. lipids and per cent. water were recorded for each genotype on the two diets.

Figs. 1 and 2 depict the patterns of change in wet and dry weight, respectively, over the 18-day period of analysis. The normal genotype on diets 0

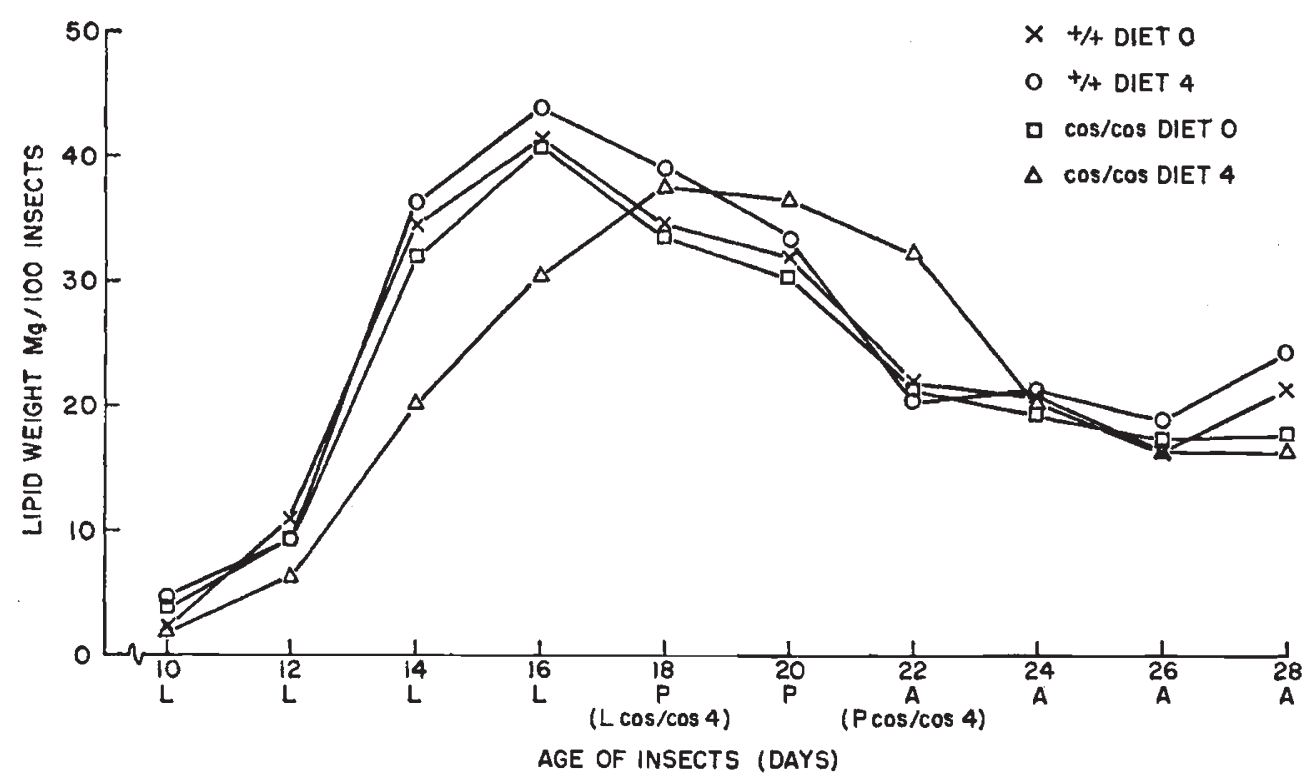

FIG. 3.-Lipid weight of experimental groups from 10 to 28 days.

and 4 and the mutant genotype on diet 0 are quite comparable throughout development. The mutant genotype on diet 4 shows a marked reduction in weight during the larval stages. An important point must be emphasised at this time. One can not accurately compare animals of the same age because morphological and physiological changes are not directly related to age. For example, the mutant genotype on diet 4 appears to be two days out of phase with the other insects and thus at day 18 the larval stage of this group is compared with the pupal stage of the other three groups. Although this consideration is important the data does support the basic hypothesis that an oil high in unsaturated fatty acids will inhibit growth of the $\cos / \cos$ genotype and has no effect or may even enhance the growth of $+1+$ genotype.

Fig. 3 is a graph of the lipid weight of the insects with the days of culture. The lipid weight increases rapidly and peaks on the last day of the larval stage. During pupation the total weight of lipids decreases, reflecting the cessation of eating and the internal mobilisation of the fatty acids needed for the morphological change to adults. The insects begin to again increase 
their lipid weight on the 28 th day suggesting that the adults, although formed on the 22nd day, do not start eating again until at least the 24th day. Again the mutant genotype on diet 4 shows the same overall pattern as the other groups but appears to be out of phase by approximately two days.

The percentage of lipids and of water in the beetles are presented in table 2. The per cent. lipids based on the dry weight of the insects varied between 18 and 49 per cent. Surprisingly, very little difference in the total per cent. lipids was observed between the insects grown on the two different diets. The total water content varied between 55 and 81 per cent. during the culture period. From the 14th day on, regardless of the morphological stage, the per cent. water was relatively constant, 56 to 63 per cent.

TABLE 2

Percentage of lipids and of water in Tribolium castaneum

\begin{tabular}{|c|c|c|c|c|c|c|c|c|}
\hline \multirow[b]{2}{*}{ Day } & \multicolumn{2}{|c|}{$+1+\operatorname{diet} 0$} & \multicolumn{2}{|c|}{$+/+\operatorname{diet} 4$} & \multicolumn{2}{|c|}{$\cos / \cos \operatorname{diet} 0$} & \multicolumn{2}{|c|}{$\cos / \cos \operatorname{diet} 4$} \\
\hline & Lipids* & Water & Lipids & Water & Lipids & Water & Lipids & Water \\
\hline $10(L) \dagger$ & 29 & 78 & 24 & 71 & 24 & 69 & 45 & 81 \\
\hline 12 (L) & 29 & 66 & 18 & 65 & 34 & 65 & 30 & 68 \\
\hline $14(\mathrm{~L})$ & 37 & 60 & 37 & 59 & 49 & 59 & 29 & 62 \\
\hline $16(\mathrm{~L})$ & 37 & 57 & 39 & 57 & 40 & 55 & 40 & 62 \\
\hline $18(\mathrm{P}, \mathrm{L})$ & 37 & 58 & 38 & 57 & 36 & 57 & 39 & 58 \\
\hline $20(P)$ & 34 & 59 & 34 & 58 & 36 & 59 & 38 & 56 \\
\hline $22(\mathrm{~A})$ & 29 & 60 & 30 & 62 & 31 & 61 & 34 & 58 \\
\hline $24(\mathrm{~A}, \mathrm{P})$ & 26 & 59 & 27 & 58 & 26 & 59 & 28 & 59 \\
\hline $26(\mathrm{~A})$ & 20 & 60 & 21 & 56 & 22 & 54 & 24 & 61 \\
\hline 28 (A) & 24 & 56 & 27 & 55 & 26 & 63 & 21 & 57 \\
\hline
\end{tabular}

* Based on dry weight of the organism.

$\dagger \mathrm{L}=$ larvae, $\mathrm{P}=$ pupae and $\mathrm{A}=$ adult, $\cos / \cos$ on diet 4 are larvae at 18 days and pupae at 24 days.

The relative percentage of fatty acids in the $+1+$ and cos/cos genotypes on diets 0 and 4 are given in tables 3 and 4 . In an earlier study (Costantino et al., 1966) the relative percentages of $\mathrm{C}_{16}-\mathrm{C}_{18}$ fatty acids were determined for the $+1+$ and $\cos / \cos$ genotypes at 13 days of age. A clear difference in the distribution of fatty acids was noted suggesting a genetic block with the resultant accumulation of linoleate. If attention is focused on day 12 (table 4) this same general trend is observed. However, examination of the distribution of fatty acids over the 18-day period of analysis does not reveal a marked difference between the genotypes. In particular after the 14th day, the fatty acid composition was similar and relatively constant in each culture. Oleic acid was most abundant (31-8-45.4 per cent.), followed by palmitic (26.8-35.0 per cent.), linoleic (9.7-30.6 per cent.), stearic (5.9-11.8 per cent.), palmitoleic (2:0-4.7 per cent.) and myristic acids ( $<1$ per cent.). Fatty acids present in amounts less than 0.5 per cent. were not included. In addition, there was no marked variation in the relative concentration of fatty acids associated with the morphological stages during development. These data seriously question our working hypothesis of a genetic block associated with the utilisation of the unsaturated fatty acids. The studies do suggest the more subtle hypothesis of enzyme induction.

The $+1+$ and the cos/cos insects grown on diet 0 possess approximately the same fatty acid composition. Consequently, the mutant does not appear 


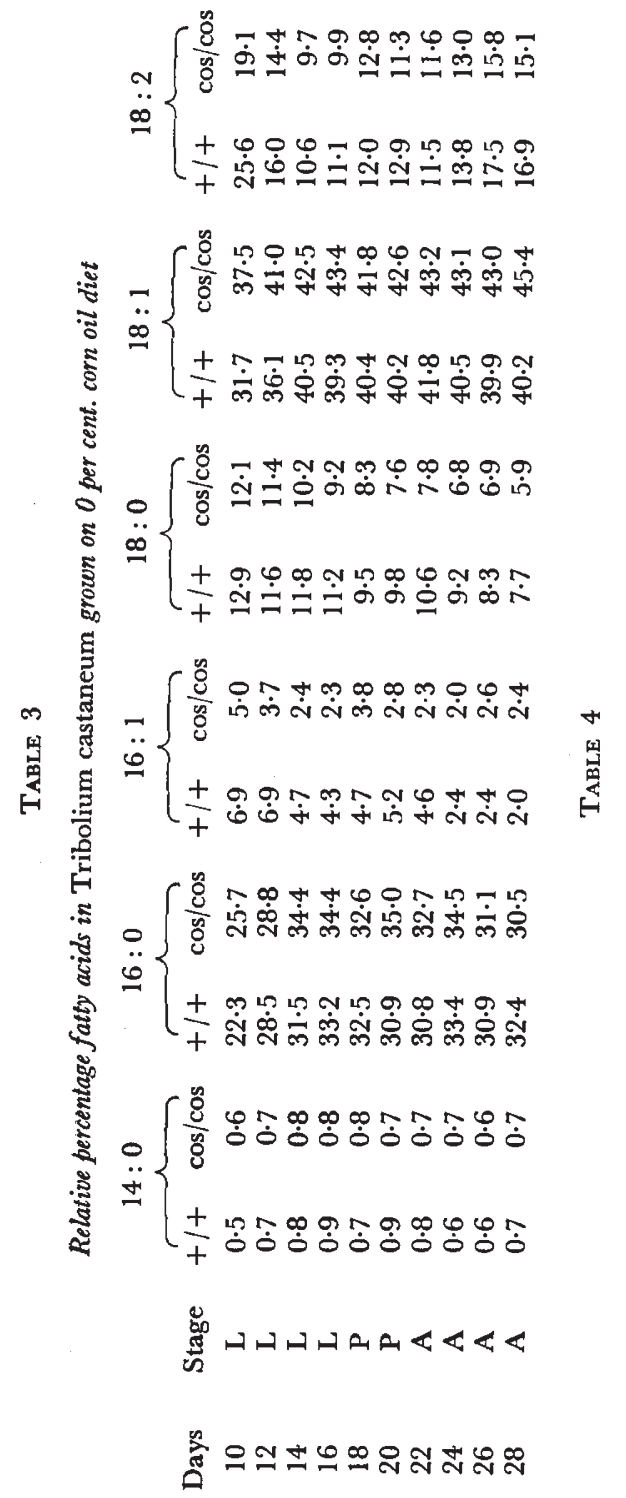

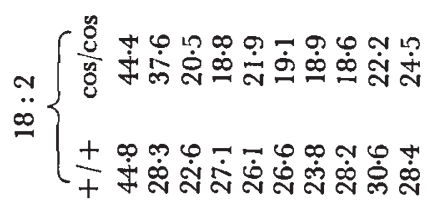

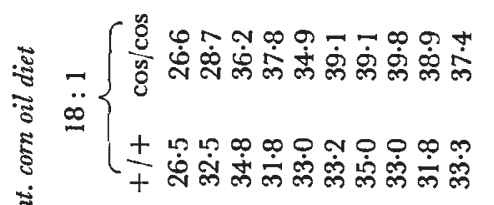

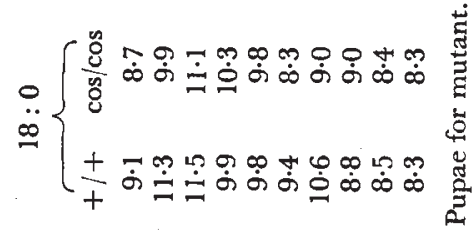

-

$\ddot{0}$

:

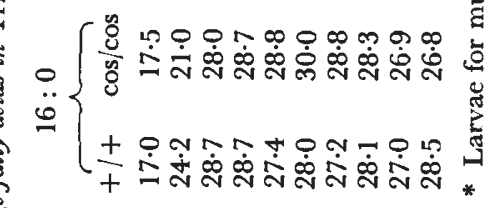

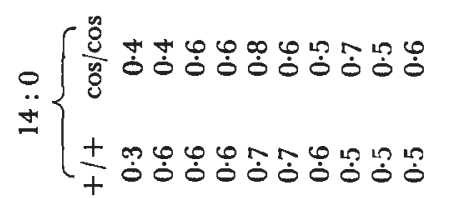

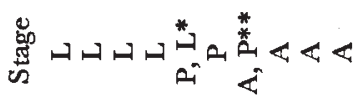

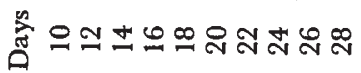


to have an unusual fatty acid metabolism when grown in the absence of corn oil.

The insects grown on diet 4 have similar but not identical fatty acid compositions. The high percentage of linoleic acid on the 10th and 12th days may reflect some surface contamination. At 10 days of age the organisms are very small; their surface area is large compared to their total weight. Although extreme care was taken to make sure the insects were clean, the 4 per cent. corn oil diet is somewhat sticky and surface contamination cannot be ruled out. This possibility is suggested when one compares the fatty acids of the insect eggs, the diets and the corn oil, table 5. The fatty acid composition of the eggs resembles the composition of the older insects (14th-28th day) grown on diet 4. Conversely, the composition of the younger insects resembles that of the corn oil diet.

TABle 5

Relative percentage of lipids and fatty acids in eggs of Tribolium castaneum, in diet 0 and diet 4 and in corn oil

\begin{tabular}{lcccccccc} 
& \multicolumn{8}{c}{ Fatty acids } \\
\cline { 2 - 8 } \multicolumn{1}{c}{ Sample } & Lipids & $14: 0$ & $16: 0$ & $16: 1$ & $18: 0$ & $18: 1$ & $18: 2$ & $18: 3$ \\
Eggs $+/+$ & 11.3 & 0.4 & 28.0 & 0.7 & 10.8 & 35.5 & 24.6 & - \\
Eggs cos/cos & 11.0 & 0.3 & 28.7 & 0.8 & 9.8 & 39.6 & 20.8 & - \\
Diet 0 & 1.3 & - & 21.6 & 6.1 & 2.6 & $14 \cdot 1$ & 53.9 & 1.7 \\
Diet 4 & 5.8 & - & 12.4 & 0.7 & 2.0 & 24.3 & 59.8 & 0.8 \\
Corn oil & - & - & 11.2 & - & 1.4 & 25.4 & 61.9 & -
\end{tabular}

The actual percentages of individual fatty acids found do not change appreciably between the 14 th and 28 th day old insects. The $+/+$ and $\cos / \cos$ genotypes differ primarily in the relative composition of oleic and linoleic acids. The normal insects possess between 31.8 and 35.5 per cent. oleic acid while the mutant has a slightly greater amount, 34.9 to 39.8 per cent. Linoleic acid is present in the $+1+$ genotype in greater amounts $(22 \cdot 6-28 \cdot 2$ per cent.) than is found in $\cos / \cos (18 \cdot 8-21.9$ per cent.) in $14-$ to 24-day-old insects. This suggests that linoleic acid is either being metabolised in the mutant at a greater rate than in the normal, or else it is not being absorbed from the diet in as large quantities as by the normal insects. Under our conditions of analysis no unusual fatty acids were detected in the mutant.

The diet influences the fatty acid composition of the insects but the diet is obviously not the determining factor. The composition of the insects is quite different from that of the diet particularly with respect to palmitic, oleic and linoleic acids.

As has been noted in the wet weight, dry weight and percentage lipids, the $\cos / \cos$ genotype (diet 4) has essentially the same composition as the normal insects, but they appear to be out of phase by approximately two days. This same observation is noted in examining the percentage of linoleic acid. These data suggest that we are dealing with an inducible enzyme system with a time lag associated with the utilisation of vegetable oils or more specifically unsaturated fatty acids. This hypothesis can explain our previous observations of (i) the differential growth pattern of the mutant, and (ii) the reduced viability of the mutant at 13 days of age but no marked lethality beyond this age. 
Since the physiological differences between the normal and mutant genotypes grown on diet 4 are already evident at 10 days of age, future work will be directed towards the analysis of the development of the younger insects. Radio-labeled linoleic acid will be used in an effort to further characterise the mutation.

\section{Summary}

1. The unsaturated fatty acid sensitive genotype ( $\cos / \cos )$ of Tribolium castaneum was compared with the normal $(+/+)$ on diets with 0 and 4 per cent. corn oil. The relative percentages of fatty acids, wet weight, dry weight, per cent. lipids and per cent. water were monitored from 10 days to 28 days of age.

2. The $\cos$ homozygote and $+/+$ genotypes on diet 0 show the same composition in all criteria examined.

3. On diet 4 the $\cos / \cos$ genotype has the same general pattern as the normal for wet weight, dry weight and percentage lipids except that the mutant is approximately 2 days out of phase. Critical to the hypothesis of a genetic block the distribution of fatty acids of both genotypes are quite similar, especially after 14 days of age, and does not reflect the altered growth pattern of the mutant.

4. The apparent initial delay in response to the unsaturated fatty acid linoleate together with a subsequent normal pattern of development suggest that the genetic mutation may be associated with an inducible enzyme system.

\section{REFERENGES}

COSTANTINo, R. F., BELL, A. E., AND ROGLer, J. C. 1966. Genetic control of lipid metabolism in Tribolium. Nature, 210, 221-222.

COSTANTINO, R. F., BELL, A. E., AND ROGLER, J. C. 1967. Genetic analysis of a population of Tribolium. I. Corn oil sensitivity and selection response. Heredity, 22, 529-539.

COSTANTINO, R. F., ROGLER, J. C., AND BELL, A. E. 1968. Genetic analysis of a population of Tribolium. II. Metabolic pattern of corn oil sensitive anomaly. Heredity, 23, 477-483. FOLCH, J., LEES, M., AND SLOANE-ST ANLEY, G. H. s. 1957. A simple method for the isolation and purification of total lipids from animal tissues. F. Biol. Chem., 226, 497-509.

MORRISON, W. R., AND SMrTh, L. M. 1964. Preparation of fatty acid methyl esters and dimethylacetols from lipids with boron fluoride-methanol. F. Lipid Res., 5, 600-608. 